\title{
APLICACIÓN DE LA GEOMÁTICA AL ANÁLISIS DE RECURSOS MARINOS PESQUEROS EN LA COSTA DE LA COMUNIDAD VALENCIANA
}

\section{APPLICATION OF GEOMATICS TO THE ANALYSIS OF MARINE FISHERIES RESOURCES ON THE COAST OF THE VALENCIAN COMMUNITY}

\author{
Pablo Pascual-Yarritu*, Gaspar Mora-Navarro, Carmen Femenia-Ribera \\ Departamento de Ingeniería Cartográfica, Geodesia y Fotogrametría, Universitat Politècnica de València, Camino de Vera s/n, 46022 \\ Valencia, España. jospasya@alumni.upv.es; joamona@cgf.upv.es; cfemenia@cgf.upv.es
}

\begin{abstract}
:
Fishing production is one of the most important economic activities in the world. It is also important at the national level as Spain ranks as one of the 25 main producing countries of marine capture fisheries (FAO 2020). Due to its importance, its impact on the volume of resources and the performance offered by current technological development, it has extended a generalized concern about the implications that overexploitation of fishery resources could have on the marine environment and on the sustainable development of species. This concern highlights the need to know the situation of marine resources, the issue that has led to the development of this research. This study seeks to understand the life cycle of a specific species, the Octopus vulgaris (octopus), by locating the areas and times of greatest and least production. For this, the data recorded about the catches of this species in the Marina Alta region, located in the north of the province of Alicante (Spain), are analyzed. On the other hand, discovering suitable areas for octopus breeding is also desired. There are already some areas dedicated to it located by the Institut de Recerca Oceanogràfica de Xàbia (IROX) and what is intended is, based on them, to analyze their marine context to find others with similar characteristics and that, therefore, could potentially be suitable for the reproduction of the species. Its finding would be a great advance in anticipating situations that compromise the sustainable development of the species. The results of the study are transferred to a geoportal where the examined variables are graphically represented. Its function is to facilitate the interpretation of the collected data and to constitute in itself a tool that allows the visualization of the movements of octopus populations over time.
\end{abstract}

Key words: geomatics, geoportal, sustainable development, fishing, Octopus, marine cadastre

\section{Resumen:}

La producción pesquera es una de las actividades económicas con más trascendencia a nivel mundial. También lo es en el ámbito nacional y es que España se sitúa como uno de los 25 principales países productores de pesca de captura marina (FAO 2020). Debido a su importancia, a su impacto sobre el volumen de los recursos y al rendimiento que ofrece el desarrollo tecnológico actual, se ha extendido una preocupación generalizada sobre las implicaciones que podría tener una sobreexplotación de los recursos pesqueros en el medio marino y en el desarrollo sostenible de las especies. Esta inquietud pone de manifiesto la necesidad de conocer la situación en la que se encuentran los recursos marinos, cuestión que ha propiciado el desarrollo de esta investigación. Este estudio busca comprender el ciclo vital de una especie en concreto, el Octopus vulgaris (pulpo), mediante la localización de las zonas y las épocas de mayor y menor producción. Para ello, se analizan los datos registrados sobre las capturas de esta especie en la comarca de la Marina Alta, situada en el norte de la provincia de Alicante (España). Por otro lado, también se quiere descubrir zonas aptas para la puesta/cría del pulpo. Ya existen unas zonas dedicadas a ello localizadas por el Institut de Recerca Oceanogràfica de Xàbia (IROX) y lo que se pretende es, a partir de ellas, analizar su contexto marino para hallar otras con características similares y que, por tanto, puedan ser potencialmente adecuadas para la reproducción de la especie. Su hallazgo supondría un gran avance para prever situaciones que comprometan el desarrollo sostenible de la especie. Los resultados del estudio se vuelcan en un geoportal donde se representan gráficamente las variables examinadas. Su función es facilitar la interpretación de los datos recopilados y constituir en sí mismo una herramienta que permita visualizar los movimientos de las poblaciones de pulpo a lo largo del tiempo.

Palabras clave: geomática, geoportal, desarrollo sostenible, pesca, Octopus, catastro marino

\section{Introducción}

El origen de este estudio es consecuencia directa de un proyecto iniciado por el Institut de Recerca Oceanogràfica de Xàbia (IROX 2015), una asociación que vive por y para el estudio científico del mar y del litoral, su protección y la divulgación de su conocimiento. Con el fin de conseguir la preservación, protección y recuperación de la biodiversidad de la Marina Alta, esta asociación ha puesto su atención en una especie con gran relevancia

‘Corresponding Author: jospasya@alumni.upv.es, jospasya@alumni.upv.es 
en la Comunidad Valenciana: el pulpo (Octopus Vulgaris).

El propósito del IROX es conocer cuál es la situación actual del pulpo en las costas de la Marina Alta. Su intención es tener un control aproximado de la especie para poder adoptar medidas ajustadas a la realidad y que fomenten la sostenibilidad de la especie y ayuden a conseguir una gestión pesquera más eficiente (ArcasSen 2019). Para ello, cuenta con la implicación de la flota tradicional del Pòsit de La Marina en la recopilación de datos sobre las capturas.

A partir del conocimiento de la existencia de este proyecto y de esos datos y de visualizar el gran potencial que la Geomática podría aportar a la consecución del objetivo del IROX, se produce el primer contacto y acaba surgiendo esta investigación.

Así pues, el empeño de este estudio pasa por aprovechar los datos que el IROX recopila sobre las capturas de pulpo para tratarlos y representarlos en una herramienta útil que facilite su accesibilidad y visualización: un geoportal.

\section{Justificación}

El proyecto del IROX tiene su base en la recopilación de los datos sobre las capturas de pulpo. Esta recopilación se traduce en un conjunto de datos que aumenta progresivamente. Los datos y su tratamiento son vitales para la consecución de sus objetivos. Los integrantes de la asociación tienen competencias en biología, pesca, buceo y otros ámbitos relacionados con el mundo marino, sin embargo, actualmente no disponen de un miembro con competencias en el tratamiento y la explotación de los datos. Por tanto, este es uno de los temas que se está cubriendo con esta investigación. De esta forma, el proyecto en sí se aborda tanto desde el aspecto técnico como desde el biológico.

Por otra parte, tras una primera revisión de los datos sobre las capturas de pulpo y tras consultar otras fuentes capaces de complementar estos datos con información geográfica, se comprendió que aplicar un enfoque cartográfico al proyecto podía contribuir en gran medida a conseguir un conocimiento más real de la situación de la especie en las costas de la Marina Alta. Saber dónde, cuándo y cuánto se ha pescado supone un gran impulso para lograr una gestión pesquera más eficaz (CalderónGil 2018).

Otro aspecto que quiere cubrir este estudio es la accesibilidad a los datos analizados. Se desea que todo usuario al que le atraiga esta materia, sea un miembro del propio IROX o cualquier otra persona con interés en el tema, pueda tener acceso directo a la información desde una herramienta web. Asimismo, los datos son representados mediante gráficas y mapas con el fin de hacer más fácil y rápida su interpretación por parte del usuario.

\section{Objetivos}

El principal objetivo de esta investigación es conseguir proporcionar el mayor valor añadido posible al proyecto del IROX a partir del conjunto de datos que esta asociación provee. Con esto, una vez se han analizado las posibilidades de la información suministrada y de otras que pueden ser de utilidad, se han definido tres objetivos específicos:

- $\quad$ Disponer de un control de las capturas de pulpo.

- Descubrir zonas aptas para la puesta de pulpo.

- Conocer cuál es la evolución espacio-temporal del pulpo.

Estos tres propósitos se encuadran dentro del marco geográfico que definen los datos de partida, que actualmente comprende la costa de la Marina Alta.

El primero de los puntos que se han señalado como objetivos consiste en tener un control de las capturas a modo de cuadro de mando o dashboard. El propósito de esto es tener una herramienta de gestión pesquera que facilite la toma de decisiones y que recoja un conjunto de indicadores que proporcionen a los usuarios una visión comprensible del estado de la pesca del pulpo en la zona analizada (Fernández-González 2009).

El siguiente objetivo en la lista trata de encontrar zonas aptas para la cría del pulpo. Desde el IROX se ha trasladado que, por su parte, se han hallado varias zonas aptas para la puesta del pulpo. La intención aquí es analizar cuáles son las características de esas zonas y, atendiendo a esto, descubrir otras con características similares que puedan ser también potencialmente aptas para la cría de la especie.

Por último, conocer cuál es la evolución espacio-temporal de las capturas del pulpo sería un gran avance para tener una visión más ajustada a la realidad sobre el movimiento de esta especie. El componente temporal está registrado en el histórico de capturas de pulpo que recopila el IROX. Sin embargo, el componente espacial está mucho menos determinado, más allá de saber a qué puerto pertenece cada embarcación pesquera.

Existen fuentes de información que proporcionan datos y servicios de localización de embarcaciones tanto en tiempo real como en series históricas. Algunas gratuitas y otras de pago.

Al enlazar esta información junto con los históricos del IROX, se daría respuesta a dónde, cuándo y cuánto se ha pescado. Esto daría un gran impulso hacia la consecución de una gestión pesquera más eficiente.

\section{Metodología}

\subsection{Descripción general}

El punto de partida de esta investigación tiene lugar a partir del conocimiento del proyecto sobre el estudio del pulpo del IROX y concretamente a partir de la revisión del conjunto de datos sobre capturas que esta organización ha ido recopilando en los últimos años. Tras el primer reconocimiento, se observa que es necesario ordenar el contenido de los datos y parametrizarlos de forma que más adelante puedan ser importados en una base de datos.

Los datos iniciales son una serie de hojas de cálculo con información sobre las capturas de pulpo, tales como la fecha, la embarcación, el puerto de origen, el volumen de pesca o el precio de mercado en ese momento, entre otros. El primer archivo proporcionado por el IROX 
contiene datos desde 2013 a 2016, aunque se han ido incorporando progresivamente los datos hasta 2021.

Como uno de los propósitos es proporcionar una herramienta web que dé accesibilidad a la información sobre capturas de pulpo, se hace esencial la creación de una base de datos. Para ello, primero se ha diseñado la estructura de la misma y más tarde se han importado los datos contenidos en las hojas de cálculo. Esto último es un proceso que se repite cada vez que se dispone de más información.

Una vez los datos han sido almacenados y están listos para ser consultados, se ha dado comienzo a la creación de la herramienta web. Esta a su vez se divide en las siguientes partes:

- Web de inicio o presentación de la investigación.

- Geoportal.

\section{- Servicio API REST.}

La web de inicio tiene el papel de introducir al usuario en la investigación. La intención es que el usuario, al navegar por ella, tenga claro cuál es el contexto global de este estudio. Para ello se han incluido varias secciones que detallan cuáles son los objetivos, el equipo de trabajo, los colaboradores o el contacto para cualquier consulta.

El geoportal, por su parte, es la parte de la herramienta web que muestra al usuario los datos sobre las capturas de pulpo. Para facilitar su interpretación, la información analizada se muestra mediante representaciones visuales como gráficos, diagramas, series, etc. Asimismo, se está desarrollando un visor cartográfico donde se incorporan diferentes capas de información geográfica con la intención de indicar cuáles son las zonas que se han seleccionado como posibles zonas aptas para la puesta de pulpo. Por último, aunque aún sin comenzar, se pretende incluir un servicio de descarga de datos.

El otro elemento de la herramienta web es el servicio API REST. Es una capa de abstracción entre el portal y la base de datos. Cuenta con varios endpoints documentados con los que poder consultar y filtrar la información sobre las capturas de pulpo registradas.

Creada ya la base de datos e implementada toda la herramienta web, queda un paso importante para conseguir que la aplicación sea accesible: su puesta en marcha. Para ello, se ha habilitado un servidor virtual donde se aloja toda la infraestructura necesaria para que la herramienta web funcione correctamente. Por otra parte, se ha elegido un dominio gratuito para evitar que los usuarios tengan que acceder a la web mediante una IP.

Todo el desarrollo web se ha añadido a un repositorio remoto con control de versiones. De esta forma, se mejora el control sobre el trabajo realizado y se minimiza la posibilidad de pérdida de trabajo en el caso de que el servidor se restaurara.

A la vez que se ha ido desarrollando toda la infraestructura digital, se ha realizado una labor constante de búsqueda de información en diferentes fuentes con el afán de complementar los datos de las capturas proporcionados por el IROX.
Una de las fuentes que se han consultado continuamente ha sido el Centro de Descargas del Centro Nacional de Información Geográfica (CNIG), desde donde ha sido posible acceder a datos con información geográfica sobre puertos, límites marítimos, línea de costa, etc. Todos ellos producidos por el Instituto Geográfico Nacional (IGN).

Otra fuente muy consultada ha sido el Instituto Español de Oceanografía (IEO), que proporciona información muy útil del entorno marino, como la batimetría, isóbatas, naturaleza del fondo marino, arrecifes, caladeros y otros límites marítimos que no se han encontrado en otras fuentes, como la zona de Mar Territorial y la Zona Contigua

El Ministerio de Agricultura, Pesca y Alimentación también cuenta con información geográfica relevante para este estudio, ya que desde su catálogo se puede acceder a las eco-cartografías por provincias. Entre su conjunto de datos destacan los usos del suelo, la morfología del fondo, la calidad de aguas y las comunidades marinas.

En cuanto a las fuentes sobre localización de embarcaciones, se han consultado varios servicios como MarineTraffic o VesselFinder que, aunque sí disponen de la información necesaria, su acceso es de pago. Existen alternativas gratuitas como AISHub, aunque no monitoriza todos los puertos deseados.

\subsection{Especificaciones técnicas}

Los tres elementos de la herramienta web, la página de inicio, el geoportal y el servicio API REST, tienen un elemento en común y es que todos ellos se han desarrollado bajo un mismo framework: Django. No obstante, para implementar el API REST se ha utilizado la librería específica Django REST Framework.

La web de inicio y el geoportal, por su lado, incluyen también implementaciones en JavaScript, aunque mucho más extensas en la parte del portal. En él, se ha empleado la librería ChartJS para la visualización de los datos en forma de gráficos y diagramas, y la librería OpenLayers para la representación de la información geográfica relacionada en forma de, por el momento, un visor cartográfico.

Las capas de información espacial incluidas en el visor se visualizan en forma de vector, a partir de datos en formato GeoJSON; o en forma de teselas o tiles a partir de WMS y WMTS.

Durante el transcurso de esta investigación, algunos servicios de información geoespacial han estado en mantenimiento, provocando un corte temporal en su servicio. Por este motivo, se ha contactado con las instituciones correspondientes para la obtención de información en ficheros SHP, especialmente de aquella que se preve estable, como las isóbatas, límites administrativos marinos, naturaleza del fondo marino, arrecifes, caladeros, etc. Posteriormente, estos archivos se han convertido a GeoJSON y se han representado como capas vectoriales en el visor cartográfico.

Por otro lado, se consumen servicios externos como el servicio de ARCGIS online de ESRI, para obtener, mediante WMTS, el mapa base World Ocean Base y sus referencias; el tileado XYZ de Open Street Maps, para 
agregar también el mapa base World OSM y algunos WMS de la Red de Información Ambiental de Andalucía (REDIAM), en cuyo catálogo existen capas con información interesante sobre límites administrativos marinos en el Mediterráneo. Sin embargo, esta última fuente de información también sufre cortes temporales en su servicio y posiblemente se deje de utilizar en el futuro.

También se ha creado información geográfica propia a partir de la obtenida desde los servicios de información geoespacial consultados. Un ejemplo son los puertos de la Comunidad Valenciana, que se encontraban dentro del conjunto de datos sobre redes de transporte del CNIG. Utilizando QGIS, se ha editado la capa y se han mantenido unicamente los puertos pesqueros, dejando fuera todos aquellos dedicados a actividades deportivas.

Otro ejemplo de creación de información geográfica son las zonas de pesca. Ante la problemática de obtener un acceso gratuito a la posición de las embarcaciones pesqueras cuando éstas ejercen su labor, se ha realizado una aproximación sobre dónde pesca cada barco un tanto grosera. Para ello, se han delimitado unas zonas de pesca atendiendo a la ubicación geográfica de los puertos de origen, a los límites de profundidad propios de cada arte de pesca (Gómez 2017) y a la linea administrativa que marca el fin de lo que se denomina Mar territorial. Asimismo se estima que, con la intención de ahorrar combustible, los barcos pescan en frente del puerto de descarga. Suposición que se considera bastante acertada según la Figura 1.

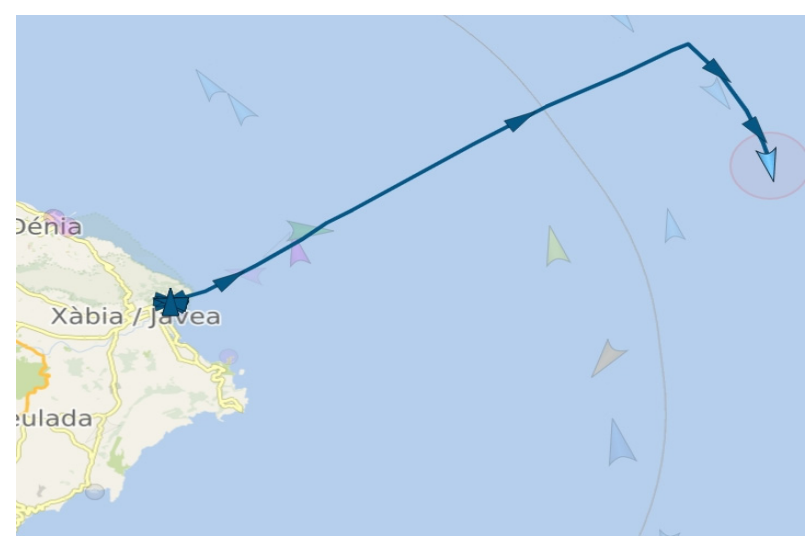

Figura 1: isualización del itinerario recorrido por uno de los barcos pesqueros desde la app VesselFinder.

Con QGIS y utilizando las capas de información geográfica relativa a los puertos, las isóbatas y la línea de Mar territorial, y tras haber corregido los errores propios de topología, se han generado ocho polígonos o zonas de pesca (Fig. 2).

Lo que se busca con estas zonas de pesca es simular, a grandes rasgos, lo que se podría conseguir teniendo la posición exacta de los barcos pesqueros y utilizando mapas de calor. Para ídentificar visualmente dónde y cuándo se ha pescado más o menos cantidad, se tienen que relacionar estas zonas de pesca con los registros de capturas. El puerto de origen de las embarcaciones y el arte de pesca de las mismas es el punto de unión.

Los datos de las capturas de pulpo están almacenados en una base de datos en PostgreSQL y sobre ella se ha añadido la extensión PostGIS. Para incluir las zonas de pesca dentro del modelo de datos se ha utilizado la herramienta shp2pgsql, que permite convertir archivos SHP en tablas PostGIS. Tras la importación, se ha realizado la relación entre las capturas y las zonas de pesca (Fig. 3).

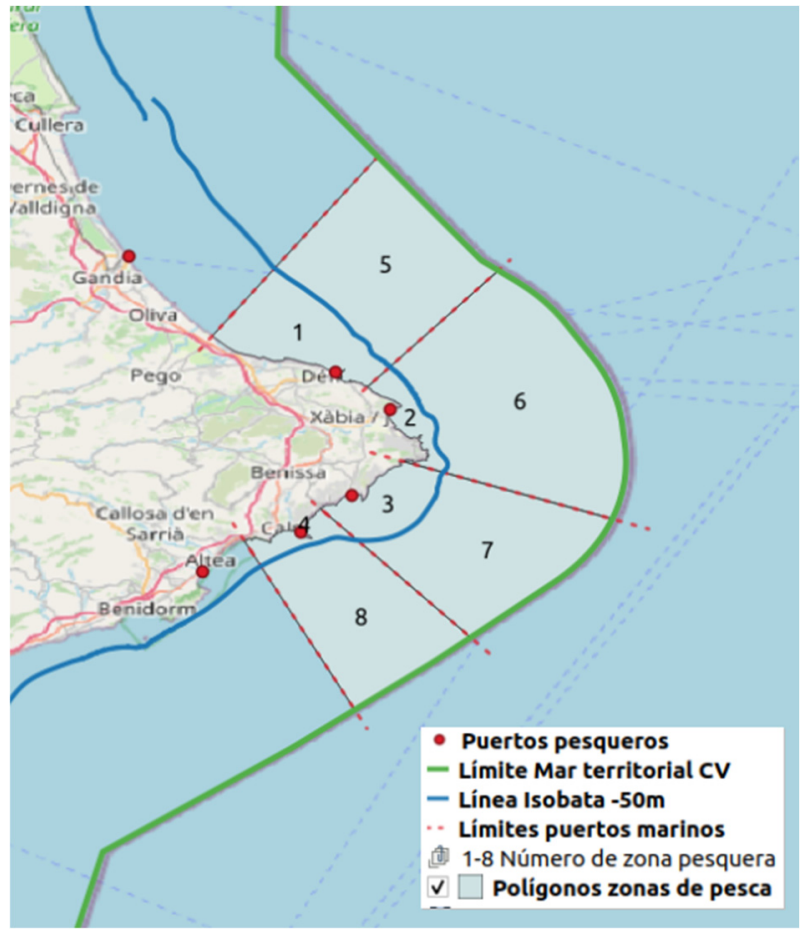

Figura 2: Delimitación de zonas pesqueras teniendo en cuenta el puerto de origen, el arte de pesca y la línea de Mar territorial.

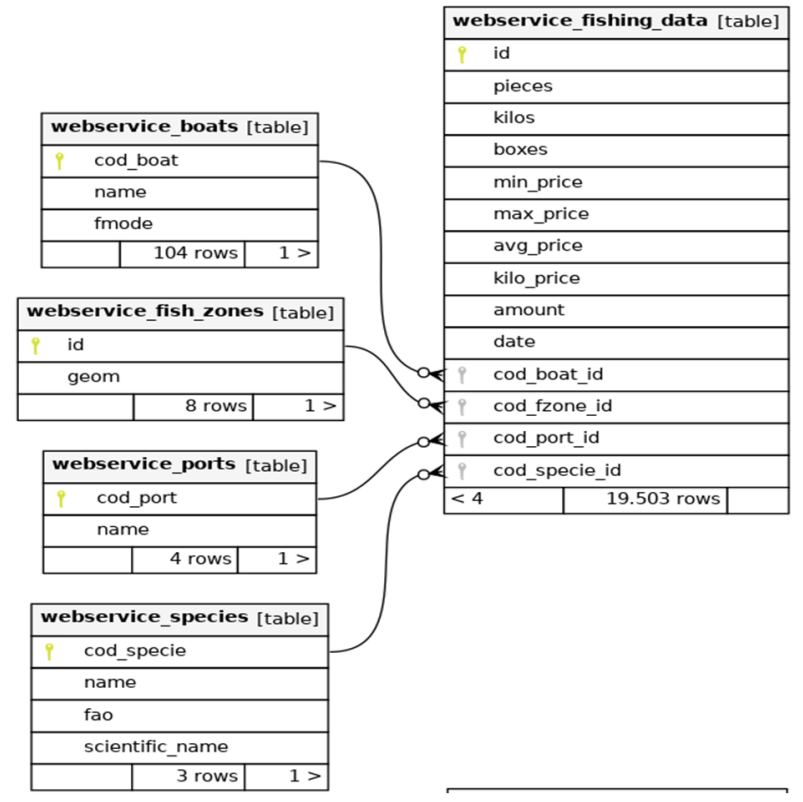

Figura 3: Diagrama entidad-relación (ERD) de los elementos principales de la base de datos.

Para la publicación de las zonas de pesca como capa de información geográfica accesible desde el visor se ha meditado utilizar GeoServer, pero finalmente se realiza desde uno de los endpoints del servicio API REST. En él simplemente se serializa el campo geometría del modelo, de forma que se convierte en un GeoJSON y queda listo para ser consumido desde OpenLayers. Las razones por la que se ha decidido publicar de esta forma y no con 
GeoServer están relacionadas básicamente con el ahorro en recursos, configuraciones e instalaciones en el servidor. Sería conveniente realizar comparaciones de los tiempos de respuesta en los dos escenarios. Como el resto de endpoints del servicio API REST, las respuestas a las consultas se guardan en caché durante un tiempo determinado en una base de datos en Redis, configurada previamente en Django REST Framework.

Toda la infraestructura tecnológica de este proyecto funciona bajo un sistema operativo Debian y se aloja en un servidor virtual de OVH. Para evitar tener que utilizar la IP del servidor para acceder a la herramienta web, se ha registrado un dominio gratuito datamar.tk. Este tipo de dominios gratuitos tienen grandes desventajas, como la dificultad de conseguir un buen posicionamiento o SEO en los principales motores de búsqueda o un ancho de banda limitado, que se traduce en un límite de tráfico mensual. La idea es seguir desarrollando la investigación de esta forma, básicamente por el tema económico, y una vez acabada o si el IROX necesita publicar sus resultados, cambiar el dominio a uno de pago. Incluso se plantea migrar la solución al propio servidor del instituto de investigación.

\section{Resultados}

El resultado visible de esta investigación es accesible desde la siguiente URL: https://www.datamar.tk/. El primer contenido que se visualiza es la web de inicio, que hace una presentación global de la investigación: objetivos, equipo, colaboradores y contacto.

Existen dos formas de avanzar al geoportal desde la web de inicio, pulsando sobre la sección correspondiente en el menú de navegación superior o apretando el botón
Acceso al Portal situado sobre el video-resumen de la cabecera. Una vez dentro se muestran cuatro bloques, cada uno de ellos relacionado con un ámbito diferente dentro del contexto de la investigación (Fig. 4). Estos son:

- Impacto económico.

- Impacto ecosistema.

- Sostenibilidad.

- Datos abiertos.

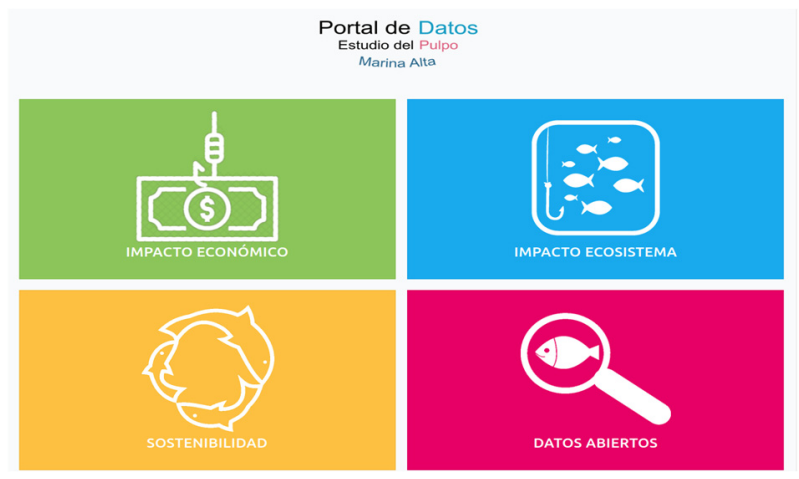

Figura 4: Bloques de contenido del geoportal.

Los dos primeros bloques contienen información acerca de las capturas de pulpo desde dos perspectivas distintas. Ambos son cuadros de mando (Fig. 5) con una estructura similar, sin embargo, el primero muestra indicadores económicos mientras que el segundo ofrece indicadores sobre el volumen de recursos marinos capturados.
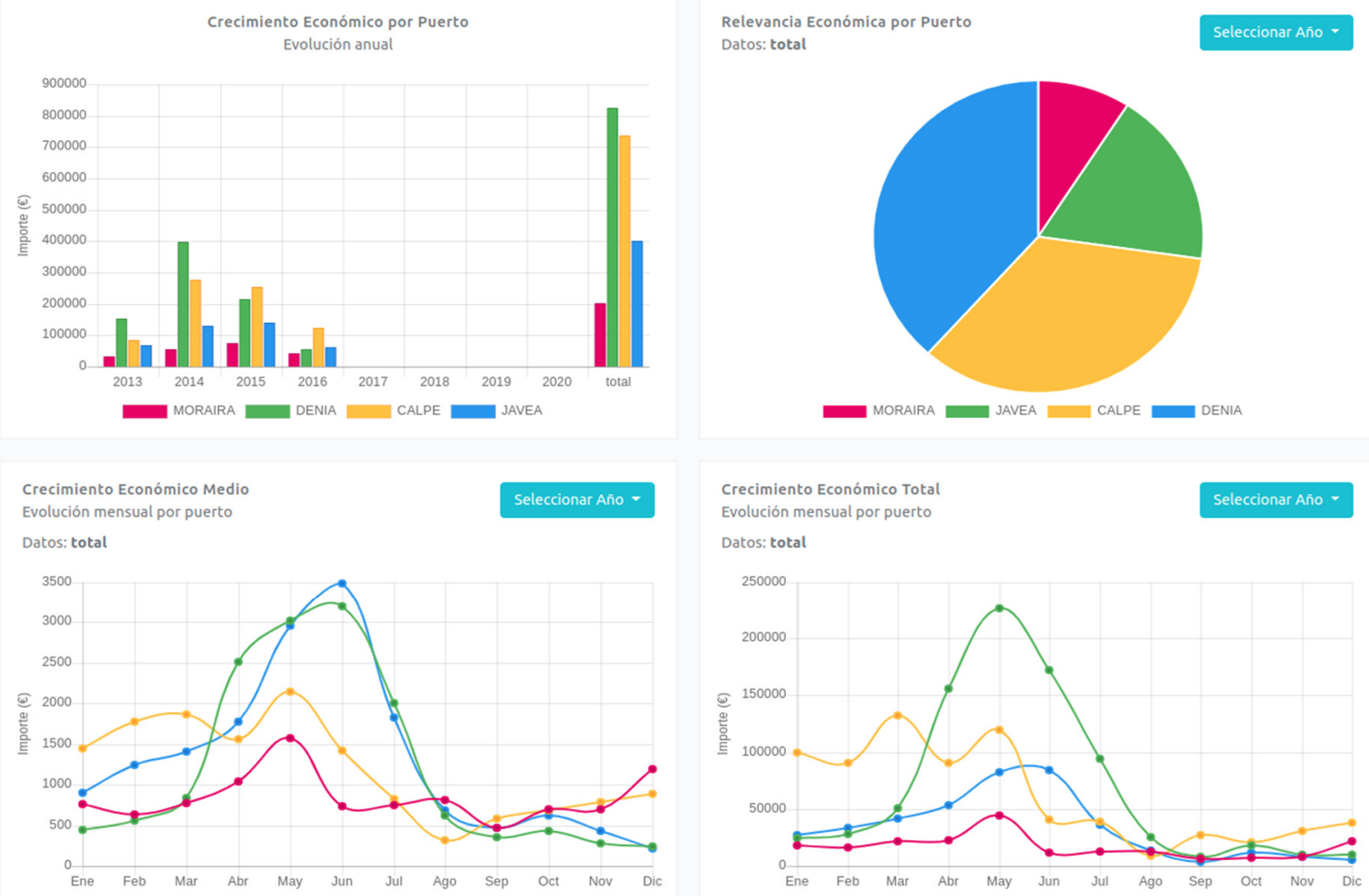

Figura 5: Fragmento de cuadro de mandos incluido dentro del bloque sobre impacto económico. 
El contenido del primer bloque trata de estimar cuál ha sido el impacto económico que ha generado la pesca de pulpo en la comarca de la Marina Alta. Es importante destacar que, aunque este proyecto trata de hacer un seguimiento de las capturas de pulpo desde 2013 a la actualidad, el componente económico se pierde a partir de mitades de 2016. Esto es el resultado de no seguir un patrón uniforme o una plantilla común a la hora de recopilar los datos de las capturas en las hojas de cálculo. De todas formas, se está tratando de recuperar esta información para poder volcar datos económicos hasta la actualidad.

Así pues, actualmente el contenido del bloque sobre el impacto económico del pulpo muestra los indicadores teniendo en cuenta las capturas realizadas entre enero de 2013 y junio de 2016. Con esto, el importe total obtenido por la pesca de pulpo durante este periodo y en los puertos de la Marina Alta es de $2.166 .823,28 €$ con un precio medio de $5,48 € / \mathrm{Kg}$.

El cuadro de mandos dispone de varias series temporales, diagramas de barras y gráficos circulares que muestran la evolución del crecimiento económico, total y promedio, y permiten hacer una comparación por año, por puerto, o por arte de pesca. Asimismo, se han incorporado un conjunto de series temporales para controlar la fluctuación media de los precios y poder compararla también por año, por puerto o por arte de pesca (Fig. 6).
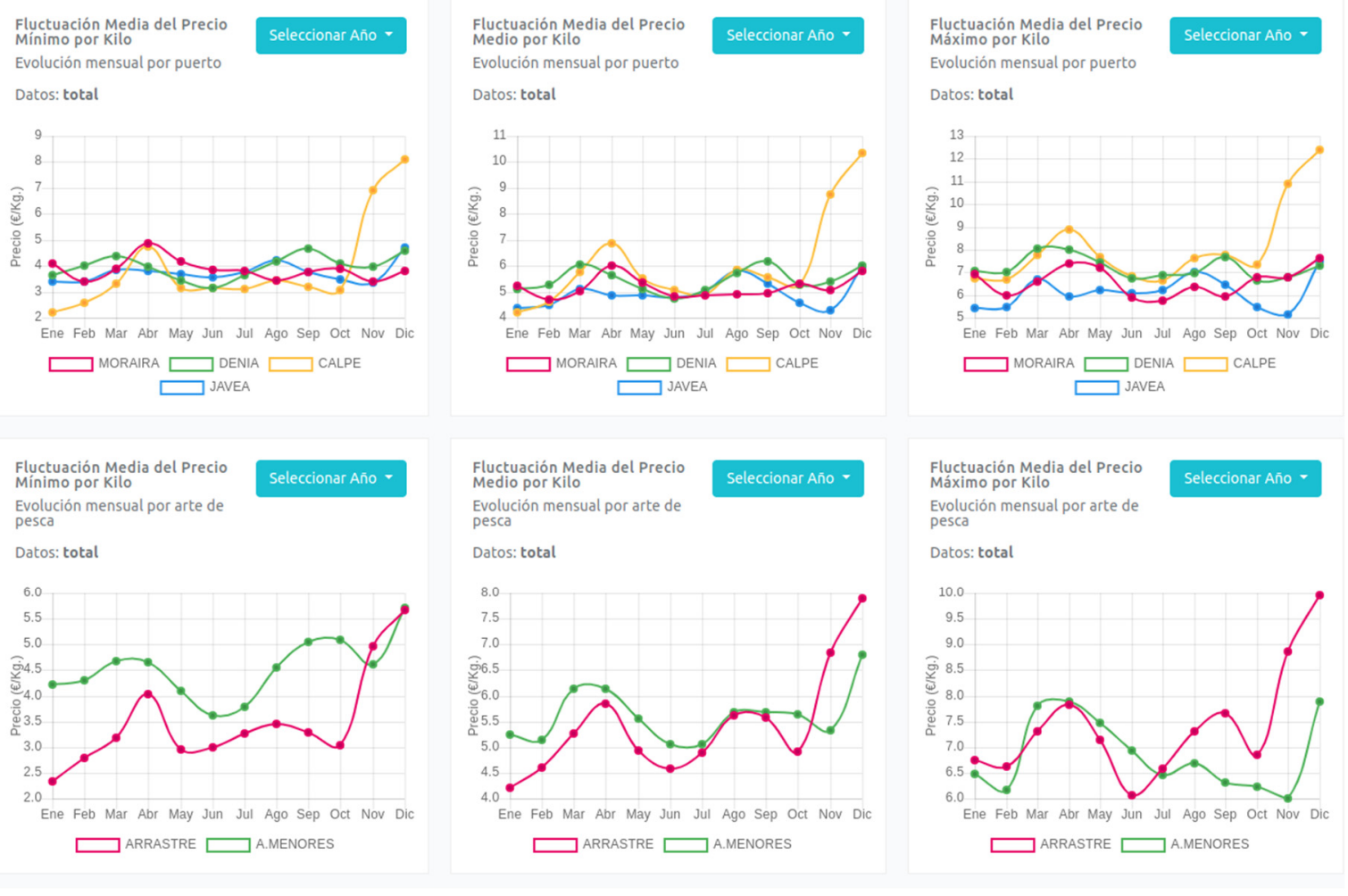

Figura 6: Control de la fluctuación media del precio por Kg. de pulpo.

Por otra parte, el contenido del segundo bloque trata de evaluar cuál ha sido el efecto de las capturas de pulpo en el ecosistema de las costas de la Marina Alta. En este caso sí que se dispone de datos de capturas desde 2013 hasta la actualidad excepto en algunos periodos en los que faltan registrar los datos de algún puerto. Esto último se está tratando de solucionar progresivamente. Así pues, el volumen de capturas total en la Marina Alta para el periodo descrito asciende a $719.779,61 \mathrm{Kg}$.

Del mismo modo que en el contenido sobre el impacto económico, los datos sobre el impacto en el ecosistema se presentan a modo de cuadro de mando mediante series temporales, diagramas de barras y gráficos circulares. Aunque esta vez en lugar de mostrar el crecimiento económico se muestra la evolución de las capturas, total y promedio. También se hace la comparación por año, por puerto y por arte de pesca.
El bloque de sostenibilidad está actualmente en desarrollo. Incluye un visor cartográfico con capas de información relacionadas con el entorno marino de las costas de la Marina Alta, como el tipo de fondo, la profundidad, arrecifes y caladeros y delimitaciones administrativas. También contiene la localización de las zonas que ya se consideran como aptas para la puesta del pulpo y se está tratando de descubrir otras nuevas para añadirlas junto a ellas en el visor. Asimismo, se han incorporado las zonas de pesca delimitadas, de forma que el usuario puede consultar el volumen de pesca en cada una de ellas para una fecha determinada (Fig. 7). También tiene la posibilidad de visualizarlo de forma secuencial mediante el botón de reproducir. El selector de capas, la información del feature seleccionado y la leyenda se encuentran en el panel lateral derecho. 


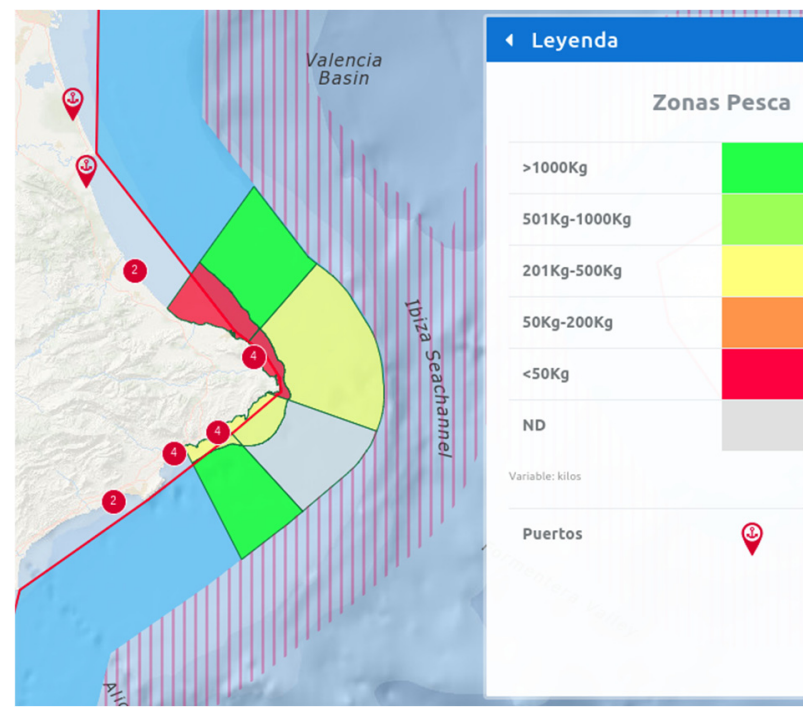

Figura 7: Volumen de capturas de pulpo por zona de pesca.

El bloque de datos abiertos no está disponible aún. La finalidad de esta sección es que los registros de capturas de pulpo de la Marina Alta puedan ser accesibles por parte de cualquier usuario interesado, siempre y cuando el IROX esté de acuerdo. De esta forma cualquier persona puede contribuir a la mejora de esta investigación e incluso puede aprovechar la información para utilizarla en futuros proyectos.

\section{Conclusiones}

Los tres objetivos planteados tanto en esta investigación como al propio IROX se encuentran en diferente situación. El punto más avanzado es el de disponer de un control de las capturas de pulpo. Actualmente ya existen dos cuadros de mandos para ello, aunque para que estos fueran más detallados se debería de completar todos los periodos en los que no se han registrado, por ejemplo, datos económicos o las capturas procedentes de puertos como Moraira o Denia.

Sin duda, el mayor problema encontrado es el de partir de unos registros de capturas con una estructura heterogénea. El primer conjunto de datos obtenido comprende desde 2013 a 2016 y en él se incluye información sobre las capturas y sus precios de mercado de los puertos de Calpe, Dénia, Moraira y Jávea. A partir de entonces, se obtienen progresivamente más registros de capturas, sin embargo, el dato económico deja de aparecer y existen periodos donde no hay información sobre capturas por parte de alguno de los puertos iniciales.

Para intentar evitar este problema en el futuro, se debe de seguir un mismo patrón en la recopilación de los datos. Actualmente el IROX los almacena en hojas de cálculo.
Sería importante que se utilizara una plantilla predefinida para ello. Esto también agilizaría en gran medida la carga de datos en el geoportal, ya que se podría crear un módulo de importación de archivos en el mismo.

Por otra parte, el descubrimiento de nuevas zonas aptas para la puesta de pulpo está actualmente en desarrollo. Se ha implementado un visor cartográfico con capas de información relacionadas con el contexto marino. Desde el IROX se ha indicado que el pulpo suele localizarse en entornos marinos con características similares que tienen relación con el tipo de suelo, la profundidad y la temperatura. Se dispone de datos sobre las dos primeras variables en la Marina Alta, sin embargo, la temperatura en el fondo marino es muy variable en muy poco espacio. La institución de Puertos del Estado proporciona datos de sensores de temperatura en el Mediterráneo, pero ninguno de ellos se localiza en la zona estudiada. Así pues, se buscarán aquellas zonas con un tipo de suelo y una profundidad similar a las zonas ya consideradas como aptas para la puesta de pulpo y se representaran dentro del visor cartográfico.

En cuanto a conocer cuál es la evolución espaciotemporal del pulpo en la Marina Alta, se ha hecho una primera aproximación con los datos originales del IROX. En ellos, el único dato que se puede tomar como referencia geográfica es el puerto de origen de las embarcaciones. Si bien, aunque la mayoría de pescadores no se alejan demasiado del puerto debido al combustible, no es un dato preciso para poder comprender el movimiento del pulpo. Se han consultado diversas fuentes que disponen de registros de posicionamiento de embarcaciones tanto en tiempo real como en históricos. Sus servicios son principalmente de pago. Obtener acceso a ellos daría un gran impulso hacia la consecución de este objetivo.

El elemento clave es saber la ruta de los barcos cada día, para poder situar las capturas diarias. De esta forma se espera poder realizar un mapa de calor animado para ver cómo se mueve la población de pulpos, y aplicar análisis geoestadístico a las variables georeferenciadas que describen las características del medio (fondo marino, profundidad, arrecifes, caladeros, etc) en las zonas donde se aprecie mayor densidad de pulpo, según las capturas, para intentar encontrar zonas similares, y tratar de comprobar en dichas zonas que efectivamente hay más población de pulpo.

\section{Agradecimientos}

Nuestro agradecimiento a los miembros del Institut de Recerca Oceanogràfica de Xàbia por impulsar este estudio y por su gran dedicación en la recogida de datos. Especialmente a Frederic Alemany porque sin su contribución no hubiese sido posible llevar a cabo este trabajo.

\section{References}

ARCAS-SEN, E., 2019. Estudio de la pesquería de pulpo común (Octopus vulgaris) en la Comunidad Valenciana. Propuestas para mejorar su gestión. Master Dissertation, Universidad de Alicante.

CALDERÓN-GIL, C., 2018. Análisis de la gestión socio-ambiental por parte de las cofradías de pescadores en la Comunidad Valenciana. Master Dissertation, Universitat Politècnica de València.

FAO. 2020. El estado mundial de la pesca y la acuicultura 2020. La sostenibilidad en acción. Roma. DOI: 10.4060/ca9229es. 
FERNÁNDEZ-GONZÁLEZ, A. M., 2009. La pesquería artesanal de Santa Pola (SE de la Península Ibérica). Identificación de tácticas de pesca y estimación de índices de abundancia. Doctoral Dissertation, Universidad de Alicante.

GÓMEZ, A., 2017. Realidad de la pesca de arrastre en el Mediterráneo: caso cofradía de pescadores San Pedro de Grao Castellón. Cátedra Tierra Ciudadana, Universitat Politècnica de València.

IROX, 2015. Institut de Recerca Oceanogràfica de Xàbia: Estudi per la recuperació de la població de polps a La Marina. Available: http://irox.cat/recerca/biodiversitat/estudi-per-la-recuperacio-de-la-poblacio-de-polps-a-la-marina/ [09/05, 2019]. 